\title{
Safety and Effectiveness of Mentor's MemoryGel Implants at 6 Years
}

\author{
Bruce Cunningham · Jonathan McCue
}

Published online: 30 May 2009

(C) Springer Science+Business Media, LLC and International Society of Aesthetic Plastic Surgery 2009

\section{Erratum to: Aesth Plast Surg}

\section{DOI 10.1007/s00266-009-9364-6}

As reported in the Patients and Methods section, at the time of the 6-year follow-up 608 patients, instead of 552 as originally reported, were evaluated, with an overall followup of $64 \%$.
As presented in Fig. 2, one of the primary reasons for explantation through 6 years was capsular contracture III/ IV at $25.6 \%$ for the Revision-Augmentation cohort and $16.4 \%$ for the Reconstruction cohort. For Augmentation and Revision-Reconstruction patients, the percentages for contracture III/IV, $19.7 \%$ and $29.4 \%$, respectively, were the same as II/III/IV, as reported in the text.

The online version of the original article can be found under doi:10.1007/s00266-009-9364-6.

B. Cunningham $(\bowtie) \cdot$ J. McCue Division of Plastic and Reconstructive Surgery, University of Minnesota Medical School, Mayo Memorial Bldg., 420 Delaware Street SE, Minneapolis, MN 55455, USA e-mail: cunni001@umn.edu 